\title{
The Role of HIV Antiretroviral Medication on Motor-Cognitive and Neurological Alterations in Hispanic People Living with HIV
}

Martín G. Rosario PT, PhD, CSFI, ATRIC ${ }^{*}$, Leah Jamison SPT ${ }^{1}$, Gabriel Gines BS, CSFI, CPT ${ }^{2}$

${ }^{1}$ Texas Woman's University, Physical Therapy Program, Dallas Campus; Texas; United States.

${ }^{2}$ Ponce Health Science University School, United States.

\author{
Article Details \\ Article Type: Research Article \\ Received date: $19^{\text {th }}$ February, 2020 \\ Accepted date: $04^{\text {th }}$ March, 2020 \\ Published date: $11^{\text {th }}$ March, 2020
}

*Corresponding Author: Martín G. Rosario, Texas Woman's University, Physical Therapy Program, Dallas Campus; 5500 Southwestern Medical Ave. Dallas, TX 75235-7299, United States. E-mail: mrosario1@twu.edu

Citation: Rosario M.G., Jamison L., \& Gines G. (2020). The Role of HIV Antiretroviral Medication on Motor-Cognitive and Neurological Alterations in Hispanic People Living with HIV. J Pub Health Issue Pract 4(1):160. doi: https://doi.org/10.33790/ jphip1100160.

Copyright: (C2020, This is an open-access article distributed under the terms of the Creative Commons Attribution License 4.0, which permits unrestricted use, distribution, and reproduction in any medium, provided the original author and source are credited.

\section{Abstract}

Motor-Cognitive alterations (MCAs) are associated with HIV infection and antiretroviral therapy. MCAs create a profound negative impact on their independence causing a burden to caregivers and the health care system. At early stages of the HIV disease, mild MCAs affecting the central nervous system can be identified in individuals with HIV, however, these MCAs increase in severity as time and the condition progress. Despite the prominence of these traits among individuals with HIV, the manifestation and severity of these MCAs are not linear.

Purpose: The aim of this project to assess the impact of medication with MCA's and comorbidities among Hispanics with HIV.

Methods: Data was collected from 575 out of 1,300 participants' record. Variables related to cd4, blood work, urinalysis, physical activity, and demographics (age, year with diagnosis) we're collected. Data analysis of this study utilizes two types of analysis univariate and bivariate for demographic profile data and to identify correlation of multiple comorbidities and antiretroviral therapy. Then a Pearson chi square was used for the relationship analysis between peripheral neuropathy and antiretroviral therapy. The majority of the subjects were male aged between 81/2- and 451/2-years old living with HIV diagnosis. Mainly, Participants were categorized as having the HIV diagnosis with a cd4 cell count of $598 \pm 346.8$ cells/uL with a nondetected viral load.

Results: showed the mains comorbidities among individuals with HIV are hypertension $28 \%$, depression $25 \%$, peripheral neuropathy $23.6 \%$. A poor relationship between peripheral neuropathy and individuals with HIV was found using between 2-3 antiretroviral therapy. However, it was not statistically significant $\left(\mathrm{X}^{2}(2)>=1.509\right.$, $\mathrm{p}=.470)$.

Conclusion: Hispanic living with HIV, MCA's manifest as depression and in some extent peripheral neuropathy. We suggest cardiovascular and psychological testing to all participants suffering with HIV. Additional studies are encouraged for strength test, cardiovascular assessment and physical activity evaluation among these subjects.

\section{Introduction}

Over 1.2 million people in the United States are living with Human Immunodeficiency Virus (HIV), a disorder that impairs the central nervous system. Antiretroviral medications can increase life by decreasing the advancement of the virus. Withal, individuals taking antiretroviral therapy also display altered motor-cognitive manifestations of the disease. Some cognitive signs are related to alterations in remembering, attention, the orientation of time and space, to carry out various tasks, and the ability to follow instructions. Besides the above, motor deficiencies can induce gait and balance alterations that can increase the risk of falls. Both cognitive and motor changes induce a profound negative impact on their independence, creating an encumbrance on caregivers and the health care system.

Never the less, many gaps in information subsist within the specific factors causing these neurologic alterations. Interestingly, the following questions may worth examining. Is there a correlation between a specific HIV medication and neurological changes? Are these antiretroviral therapy link to other comorbidities? Are we able to distinguish any of these factors and nullify or delay some cognitive and motor disorders? Do HIV medications forth with impact physical activity?

Among the 50 states, Puerto Rico is the seventh in incidence and cases diagnosed with HIV. Among these cases, young Latino males bear the highest reported cases. Taking into consideration a state like Texas is fifth among the most top states with HIV diagnosed cases and the geographic size between Texas and Puerto Rico, it is evident in Puerto Rico is a current problem that needs to be addressed.

Our aim for this inquiry was to identify the relationship between comorbidities, physical activity, HIV status, and years with HIV diagnoses with specific HIV antiretroviral medication. The specific aims are: 1) to identify the most common comorbidity, 2) explore the lipid panel profile, 3 identify the most used antiretroviral medications, and 4) to ascertain the link between neuropathy and antiretroviral medicines to best interpret the impact on health and quality of life in people living with HIV, identify specific motor-cognitive deviations and ultimately advise particular assessment and interventions to differences mentioned above.

Innovation: This research will contribute to the existing literature related to neurocognitive and neuromotor deficiencies in Hispanic people living with HIV. Additionally, this report will identify possible direct factors affecting the quality of life, and some mechanisms related to the neurocognitive and neuromotor deterioration of people living with HIV. The ultimate intention is to improve the quality of life in people living with HIV diagnosis. 


\section{Methods}

The methodological approach for this project is as follows:

1) Data were collected from participants' records. Data from all participants enrolled in La Perla de Gran Precio (LPGP) from 20002018, who had completed the Institution enrollment requirement (approximately 1,300 files), were studied/inspected for data/information completeness. LPGP participants underwent longitudinal assessments, including, but not limited to, documentation of comorbidities, medications, blood work, lipid panel, urinalysis, years with HIV diagnosis, demographics (age, gender, ethnicity), HIV status (CD4 and viral load), socioeconomic status, method of disease acquisition contagious (including sex, perinatal transmission, intravenous drugs, blood transfusions, among others), vitals and physical activity status. Most of the participants of this HIV clinic are Hispanic/Latino (in the United States); thus, this project will give us a clear profile of not only the HIV neurocognitive/motor component but, additionally, aid in the association of these elements to this ethnic group.

2) Before the data collection, a codebook was made for data insertion and mapping. After data collection, the data was organized in a database for further analysis and interpretation.

\section{Data Analysis}

For the data analysis, the demographic profile was analyzed using univariate and bivariate to know frequencies, mean, and standard deviation. Then, to answer this study's question, data analysis was conducted as follows: First, Univariate analysis was performed for frequencies of comorbidities in HIV patients. Then different comorbidities variables were categorized for correlation analysis. Second, Univariate and bivariate analyses were made to identify the frequencies of antiretroviral medication. Then the different antiretroviral drug was categorized for correlation analysis. Third, for correlation analysis between comorbidities and antiretroviral medication, a Pearson's chi-squared test $\left(\mathrm{X}^{2}\right)$ was used. The statistical program used was SPSS version 25.

\section{Results}

Data from five hundred and seventy-five out of 1300 records were collected with the complete or partial variables mentioned above. The data extraction and collection were directly retrieved from files were the comorbidities, medications, blood work, lipid panel, urinalysis, years with HIV diagnosis, demographics (age, gender, ethnicity), HIV status (CD4 and viral load) were documented.

Results in table A showed the demographic characteristics of HIV participants. Most of the participants are male among the age of 45.6 years old, with $18 \pm 8.50$ years living with HIV diagnosis. Participants were categorized as having the HIV diagnosis according to immune cell standards, see table A [cd4 $598 \pm 346.8$ cells/uL] with a non-detected viral load.

\begin{tabular}{|c|c|c|}
\hline \multirow{2}{*}{$\begin{array}{l}\text { Selected Characteristic } \\
\text { Sex }\end{array}$} & \multicolumn{2}{|c|}{ HIV Diagnostic Patient } \\
\hline & Frequencies & $\%$ \\
\hline Male & 381 & 66.6 \\
\hline Female & 191 & 33.4 \\
\hline Total & 572 & 100 \\
\hline \multicolumn{3}{|l|}{ Age (Groups) } \\
\hline \multicolumn{3}{|l|}{$45.5 \pm 11.3$ years old } \\
\hline $18-34$ & 102 & 17.2 \\
\hline $35-50$ & 279 & 48.5 \\
\hline $51-65$ & 175 & 30.4 \\
\hline 66 and over & 19 & 3.3 \\
\hline Total & 575 & 100 \\
\hline \multicolumn{3}{|l|}{ Cause of Infection } \\
\hline Contact Heterosexual & 202 & 37.4 \\
\hline Contact Homosexual & 192 & 35.6 \\
\hline Contact Homosexual/Heterosexual & 3 & 0.6 \\
\hline Intravenous drug use & 80 & 14.8 \\
\hline Blood Transfusion & 11 & 2.0 \\
\hline Perinatal Transmission & 5 & 0.9 \\
\hline Prenatal Transmission & 3 & 0.6 \\
\hline Unknow & 25 & 4.6 \\
\hline Prostitution & 1 & 0.2 \\
\hline Other & 18 & 3.3 \\
\hline Total & 540 & 100 \\
\hline \multicolumn{3}{|l|}{ CD4 } \\
\hline \multicolumn{3}{|l|}{ CD4 count $598 \pm 346.8$ cells/uL } \\
\hline $0-200$ & 58 & 10.6 \\
\hline $201-500$ & 194 & 35.6 \\
\hline 501- and over & 293 & 53.8 \\
\hline Total & 545 & 100 \\
\hline
\end{tabular}




\begin{tabular}{|l|l|l|}
\hline Viral Load & 289 & 57.7 \\
\hline Not Detected & 212 & 42.3 \\
\hline Detected & 501 & 100 \\
\hline Total & & \\
\hline
\end{tabular}

Table A. Demographic profile Frequencies (with percentage) of HIV patients from a community center focused on HIV

This study's results revealed the three most common comorbidities of these participants, hypertension $(28 \%)$, depression $(25 \%)$, and neuropathy $(23.6 \%)$ (see table B) in that order. Since hypertension was the number one comorbidity and HIV could cause hyperlipidemia leading to excessive amounts of accumulation of lipids, especially in the upper back, a condition called buffalo's hump [1], we further explore the levels of lipids in these participants. Results showed, related to the lipid panel profile, most of the participants, $76.5 \%$, exhibited average lipid values (see table C).

\begin{tabular}{|l|l|l|}
\hline Selected Characteristic & \multicolumn{2}{|c|}{ HIV Diagnostic Patient } \\
\hline Comorbidities & Frequencies & $\%$ \\
\hline Hypertension & 130 & 28 \\
\hline Chronic Pain & 80 & 16.1 \\
\hline Neuropathy & 117 & 23.6 \\
\hline Depression & 124 & 25 \\
\hline Diarrhea & 16 & 3.2 \\
\hline Hyperlipidemia & 94 & 19 \\
\hline Arthritis & 23 & 4.6 \\
\hline Anemia & 20 & 4.0 \\
\hline Diabetes & 50 & 10 \\
\hline Other Diagnostics & 109 & 22 \\
\hline
\end{tabular}

Table B. Multiple comorbidities Frequencies (with percentage) of HIV patients from a community center focused on HIV

\begin{tabular}{|c|c|c|}
\hline \multirow{2}{*}{$\begin{array}{l}\text { Selected Characteristic } \\
\text { Cholesterol }\end{array}$} & \multicolumn{2}{|c|}{ HIV Diagnostic Patient } \\
\hline & Frequencies & $\%$ \\
\hline \multicolumn{3}{|c|}{ Cholesterol $174 \pm 41.4 \mathrm{MG} / \mathrm{DL}$} \\
\hline Normal & 267 & 76.5 \\
\hline High & 82 & 23.5 \\
\hline Total & 349 & 100 \\
\hline \multicolumn{3}{|l|}{ Triglycerides } \\
\hline \multicolumn{3}{|c|}{ Triglycerides $167 \pm 124.8 \mathrm{MG} / \mathrm{DL}$} \\
\hline Normal & 246 & 75.9 \\
\hline High & 24.1 & 24.1 \\
\hline Total & 324 & 100 \\
\hline
\end{tabular}

Table C. Lipid panel Frequencies (with percentage) of HIV patients from a community center focused on HIV

\begin{tabular}{|c|c|c|}
\hline \multirow{2}{*}{$\begin{array}{l}\text { Selected Characteristic } \\
\text { Multiple Medication }\end{array}$} & \multicolumn{2}{|c|}{ HIV Diagnostic Patient } \\
\hline & Frequencies & $\%$ \\
\hline $0-1$ & 138 & 31.4 \\
\hline $2-3$ & 276 & 62.7 \\
\hline 4 or more & 26 & 5.9 \\
\hline Total & 440 & 100 \\
\hline \multicolumn{3}{|l|}{ HIV Medications } \\
\hline Norvir & 138 & 30 \\
\hline Truvada & 161 & 35.1 \\
\hline Reytaz & 80 & 17.4 \\
\hline
\end{tabular}

Table D. Multiple antiretroviral medication Frequencies (with percentage) of HIV patients from a community center focused on HIV 
Antiretroviral medication is a controversial topic since HIV to this day [2]. Since the virus can mutate, identifying a specific successful cocktail to reduce the viral load, or copies of the virus in the body is a challenge [2]. Like any other medication, antiretroviral meds come with side effects. Therefore, to better understand the impact of antiretroviral medication, this study gathers all the medicine treating HIV for each participant. In our study, participants $(n=575)$ reported 35 different medications for the treatment of HIV. Most of them (63\%), mentioned using at least one antiretroviral medication (see table D). Specifically, thirty-five percent of participants used Truvada, whereas thirty percent used Norvir as HIV therapy treatment.

Finally, when correlating the relationship of PN with antiretroviral medication, results showed a link connecting participants using between 2-3 antiretroviral medication. However, no association was found among HIV medication and PN $\left(\mathrm{X}^{2}(2)>=1.509, \mathrm{p}=.470\right)$ (see table E)

\begin{tabular}{|c|c|c|c|}
\hline \multicolumn{4}{|c|}{ Selected Characteristic } \\
\hline \multicolumn{4}{|c|}{ Multiple Medication } \\
\hline \multicolumn{4}{|c|}{$X^{2}(2)>=1.509, p=.470$} \\
\hline \multirow[t]{2}{*}{ Multiple Medication } & \multicolumn{3}{|c|}{ Peripheral Neuropathy } \\
\hline & Positive & Negative & Total \\
\hline $0-1$ & $28(27.5)$ & $93(31.5)$ & $121(30.5)$ \\
\hline $2-3$ & $65(63.7)$ & $185(62.27)$ & $250(63)$ \\
\hline 4 or more & $9(8.8)$ & $17(5.8)$ & $26(6.5)$ \\
\hline
\end{tabular}

\section{Discussion}

This study had the objective to respond to several questions. Foremost, is there a correlation between specific HIV medication and neurological alterations? Second, can antiretroviral therapy increase the risk of having or causing other comorbidities? Third, can HIV medications directly impact physical activity? Moreover, finally, with a well-defined profile of cd4 count, lipid panel values, blood work, comorbidities, among other variables, are we able to distinguish any of the factors that alter neurocognitive and neuromotor status?

Antiretroviral Medications: In this work, participants reported 35 different medications for the treatment of HIV. Most of them (63\%) reported using at least one antiretroviral medicine. Thirty-five percent of participants used Truvada, whereas thirty percent used Norvir as HIV therapy treatment. This study gives a more unobstructed perspective of the medication profile; withal, it is still unknown the impact of this therapy, side effects, in physical activity besides the cardiac alterations. Some common side effects of these medications are built up lactic acid in blood, liver problems, weakness or tiredness, muscle pain, and fast or abnormal heartbeat (www.aidsinfo.nih.gov). Nevertheless, we concluded that keeping track of the medication profile of HIV participants is essential for a full medical/side effect description.

Comorbidities and Physical Activity: Since participants with HIV exhibit balance impairments $[3,4]$ we wanted to correlate HIV medication and neurological alterations like peripheral neuropathy. The most common comorbidities of these participants were hypertension, depression, and neuropathy. These study results propose a cardiovascular deconditioning/adjustment in these participating lipid values can alter cardiac function, and also, since HIV participants often suffer from hyperlipidemia due to meds, we examine the lipid panel of these participants. The lipid panel profile of most of the participants, $76.5 \%$, showed average lipid values. Thus, this work reveals that HIV in these participants does not affect the lipid profile. Therefore, cardiac deconditioning might be due to other factors. We can hypothesize a musculoskeletal weakness and decondition, causing physical inactivity and hence the HBP phenomenon. Also, it is a possibility that the antiretroviral medication is affecting the heart pumping efficiency or the extensibility of the vascular vessels. Cardiovascular testing data (Ross protocol) is currently collected for further analysis to have a clear view of the cardiovascular profile of these participants. We advocate an increase in physical activity to the established by the American Heart Association or CDC, 180 minutes J Pub Health Issue Pract

Volume 4. 2020. 160 a week of light to moderate activity (www.cdc.org). However, additionally, we recommend submaximal cardiovascular testing to all participants with an HIV diagnosis. Another recommendation is a weekly strengthening exercise program for these participants. In future record data collection, we plan to gather strength assessment data to understand and establish a more accurate muscle strength profile and its role in physical activity.

Neurocognitive Alterations: Another aim of this study was to identify the link between HIV and motor-cognitive deficiencies. HIV reproduces within the brain's immune cells, causing inflammation and progressive cognitive and behavior changes. These neurological disorders have been attributed to the disturbance of front striatal systems [5-8], or to diffuse white matter abnormalities, as noted in MRI studies [9-11]. Among the neurocognitive impairments affecting people with HIV, there are declines in retention, logical thinking, judgment, problem-solving, and changes in personality. These disorders could interfere with everyday activities and are linked to a decrease in quality of life, inflicting strains on caregivers, and the healthcare system. In advanced cases, this neurological alteration can cause HIV dementia. In our study, the primary neurocognitive alteration in the participants is depression (25\%). As previously stated by M.S. Bhatia and Sahil Munjal (2014), depression is a severe complication in patients with HIV/AIDS Leading to physical inactivity affecting the quality of life. We urge early psychological appraisal and therapy to participants with HIV. One of the main suggested tools for healthcare professionals working with HIV is the HIV dementia scale (HDS). The HDS is a validated instrument consisting of four items with a maximum score of 12 points designed to assess memory registration, psychomotor skills, motor speed, and memory recall. According to Sacktor NC et al. [12], a score below ten can point to early signs of dementia in participants.

Neuromotor Alterations: Additionally, among HIV diagnosed individuals, most cases $(>50 \%)$ of HIV-associated motor-cognitive alterations (MCNAs) experience asymptomatic impairment, characterized by neuropathologic abnormalities and heightened risks of early mortality. Approximately $20-40 \%$ of diagnosed individuals with HIV-associated MCNAs (equating to $5-20 \%$ of all individuals with diagnosed HIV) experience mild MCNAs, and $1-2 \%$ of individuals diagnosed with HIV experience the most severe form of HIVassociated MCNAs, HIV-associated dementia [13]. According to those mentioned above in this study, we had an unexpected finding related to peripheral neuropathy (PN). Only a small percent in this study's 
samples hold PN as comorbidity, 117 out of 575. Among these subjects, further analysis reveals no link with a specific medication, diabetes, or a detected viral load, meaning the HIV medication, HIV, nor diabetes are the cause of such phenomenon, PN. When further exploring the relationship of PN with a combination of antiretroviral medication results showed a link between participants using between 2-3 antiretroviral medication and PN, however, not significant. This study concluded that neuromotor alterations are not firmly related to PN, Diabetes, or HIV due to reduce-undetected viral load among the 575 database recorded subjects.

Currently, we are exploring some other possibilities, for instance, a specific combination of HIV meds, the duration with HIV in the host's body, and gender related. Even so, the question remains, which factor(s) is causing PN and balance instability in these participants? Regardless of the cause or mechanism, we recommend monofilament for neuropathy examination among HIV diagnosed individuals. The self-perception of balance (ABC scale) is another instrument that provides valuable information, potentially revealing any selfperceived balance alteration while walking or standing in these participants [14].

\section{Limitations of the study}

This study presented several limitations; for instance, the data were collected from hard copy files. These files are stored in a room where weather factors are compromising the structural integrity of the files, making it challenging to read the record. Second, at first, ten years ago, all data (variables collected in this study) were not required to participate in the program; thus, data was missing. Third, some subjects participated during a short period, not enough to complete the full paper/document requirements, hence, incomplete data. Finally, although this study sample represents a large proportion of Latino/ Hispanics in Puerto Rico, we recognized the necessity of expanding the samples size to other territories with the same ethnicity.

\section{Conclusions}

HIV causes a burden in people living with the disease as well as caregivers. This study had the goal of further understanding the neuromotor and neurocognitive alteration than negatively impact the quality of life in these people. Also, this study discovered a weak correlation between specific HIV medication and neurological changes. Antiretroviral therapy appeared as a nonfactor on the augmented risk of having or causing other comorbidities. However, HIV patients have multiple comorbidities. Thus, it is critical to evaluate physical activity due to its apparent relation with neuromotor and neurocognitive alterations. This report identified other factors altering neurocognitive and neuromotor status, such as depression and neuropathy.

As Health Care Professionals we should recognize the importance of this type of research. HIV related study is of the utmost importance due to the risk factors related to the HIV population. As the HIV diagnosis time frame increases, so are the clinical manifestations impacting the quality of life and increasing mortality. If these altered motor-cognitive factors are evaluated and techniques are implemented to prevent or minimize the risk, we can improve the quality of life of these patients.

\section{Acknowledgement}

We graciously recognized the assistance of Ruby A. Serrano Rodríguez during this work

\section{Funding: Not Applicable}

Conflicts of interest/Competing interests: Authors report no conflict or competing interest.

Ethics approval: IRB approval TWU protocol \# 20092.

Authors' contributions: All authors contributed to the study conception and design.

J Pub Health Issue Pract

Volume 4. 2020. 160

\section{References}

1. Ceccarelli, G., d'Ettorre, G., Marchetti, F. (2011). Development of Buffalo Hump in the course of antiretroviral therapy including raltegravir and unboosted atazanavir: a case report and review of the literature. J Med Case Rep. 5:70. Published 2.

2. Esber, A.L., Coakley, P., Ake, J.A., Bahemana, E., Adamu, Y., Kiweewa, F., Maswai, J., Owuoth, J., Robb, M.L., Polyak, C.S., \& Crowell, T.A. (2020). Decreasing time to antiretroviral therapy initiation after HIV diagnosis in a clinic-based observational cohort study in four African countries. J Int AIDS Soc, 23(2) p. e25446

3. Rosario, M. G., Quiles, N., \& Ortiz, A. (2018). Balance as an assessment of health-related quality of life in people living with HIV. Journal of Human Sport and Exercise. doi: doi:https://doi. org/10.14198/jhse.2019.142.20.

4. Jamison, L. R., Gines, G., Marshall, J., \& Rosario, M. G. (2019). Vestibular and Proprioceptive Alteration Influence Postural Instability During Dual Tasks in Adults Diagnosed with HIV [Texas Woman's University]. Journal of Student Research.

5. Chang, L., Tomasi, D., Yakupov, R., Lozar, C., Arnold, S., \& Caparelli, E. (2004). Adaptation of the attention network in human immunodeficiency virus brain injury. Annals of Neurology, 56, 259-272.

6. Paul, R. H., Yiannoutsos, C. T., Miller, E. N., Chang, L., Marra, C. M., \& Schifitto, G. (2007). Proton MRS and neuropsychological correlates in AIDS dementia complex: Evidence of subcortical specificity. Journal of Neuropsychiatry and Clinical Neuroscience, 19(3), 283-292.

7. Sclar, G., Kennedy, C.A., Hill, J.M., \& McCormack, M.K. (2000). Cerebellar degeneration associated with HIV infection [letter] Neurology, 54:1012-1013.

8. von Giesen, H.J., Wittsack, H.J., Wenserski, F., Koller, H, Hefter. H, \& Arendt, G. (2001). Basal ganglia metabolite abnormalities in minor motor disorders associated with human immunodeficiency virus type 1 . Archives of Neurology. 58:1281-1286.

9. Archibald, S.L., Masliah, E., Fennema-Notestine, C., Marcott,e T.D., Ellis, R.J., \& McCutchan J.A. (2004). Correlation of in vivo neuroimaging abnormalities with postmortem human immunodeficiency virus encephalitis and dendritic loss. Archives of Neurology 61:369-376.

10. Ernst, T., Chang, L., Witt, M., Walo,t I., Aronow, H., LeonidoYee, M. (1999). Progressive multifocal leukoencephalopathy and human immunodeficiency virus-associated white matter lesions in aids: magnetization transfer mr imaging. Radiology. 210:539-543.

11. Heindel, W.C., Jernigan, T.L., Archibald, S.L., Achim, C.L., Masliah, E., \& Wiley, C.A (1994). The relationship of quantitative brain magnetic resonance imaging measures to neuropathologic indexes of human immunodeficiency virus infection. Archives of Neurology 51:1129-1135.

12. Sacktor, N.C., Wong, M., \& Nakasujja, N. (2005). The International HIV Dementia Scale: a new rapid screening test for HIV dementia. AIDS 19:1367-74

13. Woods, S.P., Moore, D.J., \& Weber, E. (2009). Neuropsychol Rev 19: 152. https://doi.org/10.1007/s11065-009-9102-5

14. Heaton, R. K., Grant, I., Butters, N., White, D. A., Kirson, D., \& Atkinson, J. H. (1995). The HNRC 500-neuropsychology of HIV infection at different disease stages. HIV neurobehavioral research center. Journal of the International Neuropsychological Society, 1(3), 231-251. 